\title{
Multiple sclerosis following bariatric surgery: A report of five cases
}

\section{Foziah Alshamrani ${ }^{1}$, Leenah Turkistani ${ }^{2}$, Nourah AlAmri ${ }^{2}$, Anwar AlZahrani ${ }^{2}$, Abdulla Alsulaiman ${ }^{1}$,} Fahad AlKhamis ${ }^{1}$, Majed AlAbdali ${ }^{1}$, Danah AlJaafari ${ }^{1}$, Aisha AlBaker ${ }^{1}$, and Abdullah AlAmri ${ }^{1}$

1. Department of Neurology - Imam Abdulrahman Bin Faisal University, Saudi Arabia 2. College of Medicine - Imam Abdulrahman Bin Faisal University, Saudi Arabia

\section{CASE SERIES}

Please cite this paper as: Alshamrani F, Turkistani L, AlAmri $\mathrm{N}$, AlZahrani A, Alsulaiman A, AlKhamis F, AlAbdali $M$, AlJaafari D, AlBaker A, AlAmri A. Multiple sclerosis following bariatric surgery: A report of five cases. AMJ 2019;12(12):336-343.

https://doi.org/10.35841/1836-1935.12.12.337-343

\section{Corresponding Author:}

Leenah Nabil Turkistani

College of Medicine - Imam Abdulrahman Bin Faisal

University, Saudi Arabia

Email: Dr.leenabil@gmail.com

\section{ABSTRACT}

Multiple sclerosis (MS) is a debilitating chronic inflammatory demyelinating disease that affects the central nervous system. The underlying cause of multiple sclerosis is unknown, yet there are various predisposing factors. Currently, bariatric surgery is an increasingly common solution for obesity. It has been reported that bariatric procedures result in miscellaneous neurological complications. To the best of our knowledge, this is the first case series to report the occurrence of MS following bariatric surgery. Here we report the cases of five patients who were recruited for inclusion following neurological examination, complete cerebrospinal fluid (CSF) study, enhanced cranial as well as spinal cord magnetic resonance (MR) images. Diagnosis of multiple sclerosis was made based on McDonalds criteria (2010). All cases were diagnosed with relapsing-remitting form of multiple sclerosis (RRMS) after various types of bariatric surgeries.

\section{Key Words}

Obesity, multiple sclerosis, bariatric surgery, relapsingremitting form of multiple sclerosis (RRMS)

\section{Implications for Practice:}

\section{What is known about this subject?}

Multiple sclerosis (MS) is a debilitating chronic inflammatory demyelinating disease that affects the central nervous system.

\section{What new information is offered in this case study?}

To notify clinician that multiple sclerosis can occur often in patients after bariatric procedures.

3. What are the implications for research, policy, or practice?

There has been an increase in the number and variety of neurological complications following bariatric surgery, which corresponds with the increasing popularity and use of bariatric surgery.

\section{Background}

Obesity is defined as a body mass index (BMI) $\geq 30 \mathrm{~kg} / \mathrm{m}^{2}$. It is a chronic condition that has become a global health concern due to its important sequelae and complications. ${ }^{1}$ Conservative treatment has shown limited effectiveness in achieving meaningful weight loss; therefore, bariatric surgery has become increasingly popular and widely utilized. $^{2}$ A survey by The international federation for the surgery of obesity and metabolic disorders (IFSO) estimated that the worldwide number of bariatric procedures has increased by more than 10 -fold in the past two decades. ${ }^{3-6}$ There is a concomitant increase in the associated complications however, there is currently a dearth of reports of worsening multiple sclerosis (MS). ${ }^{7-8}$

MS is a chronic inflammatory demyelinating disease that affects the central nervous system and causes disability, bringing with it significant social and economic consequences. ${ }^{9}$ Its worldwide prevalence exceeds two million people. ${ }^{9,10}$ Manifestations upon presentation include but are not limited to, visual disturbances as well as sensory, motor, and autonomic dysfunctions. ${ }^{10,11}$ The underlying cause of MS is unknown; however, it is 
associated with various predisposing factors. Herein, we present five cases of MS following bariatric surgery which showed that MS did occur more often after this surgery than expected.

\section{Case details}

A total of five cases were identified (Table 1).

\section{Case 1:}

A 31-year-old male had undergone bariatric surgery (bypass) in 2014. Three years later, after losing $80 \mathrm{~kg}$ (176lbs.), he presented with sudden painful blurriness of vision in his right eye associated with fatigue and imbalance for a period of two weeks. The symptoms improved after a five-day course of intravenous methylprednisolone therapy. Neurological examination revealed a visual acuity of finger count at $20 \mathrm{~cm}$ in the right and 20/16 in the left, right RAPD. His clinical characteristic were as follow: normal muscle bulk and tone, power was $4 / 5$ in the bilateral distal upper limbs, deep tendon reflexes were $3+$ and symmetric at the biceps, triceps, knees, and ankles. His expanded disability status scale (EDSS) score was three at presentation. Laboratory tests were normal. Cerebral magnetic resonance imaging (MRI) revealed multiple hyperintense lesions in the periventricular white matter characteristic of MS (Figure 1).

\section{Case 2}

A 34-year-old male had undergone bariatric surgery (laparoscopic sleeve gastrectomy) in 2015. A year later after losing $64 \mathrm{~kg}(141 \mathrm{lbs})$, he presented with right eye diplopia and ascending lower limb numbness and weakness that suggested transverse myelitis. His symptoms improved after a three-day course of intravenous methylprednisolone therapy. Neurological examination revealed sixth cranial nerve palsy of the right eye, proximal lower limb weakness $(4 / 5)$, and reflex asymmetry with the left reflexes being $3+$. Sensory examination showed diminished pinprick, position, and vibration on the left side of his body. His EDSS score was three at presentation. Laboratory tests were normal. Brain and spine MRI with contrast revealed multiple T2 and FLAIR high signal intensities involving the juxtacortical white matter and the right aspect of the medulla oblongata with evidence of gadolinium enhancement, which indicated characteristic MS lesions (Figure 2).

\section{Case 3}

A 29-year-old male with a positive family history of MS underwent bariatric surgery (laparoscopic sleeve gastrectomy) in 2014. Two years after losing 56kg (123lbs), he presented with bilateral lower limb weakness and numbness associated with urinary incontinence lasting for three days. He showed slight improvement after a five day course of intravenous methylprednisolone therapy. Neurological examination showed intact cranial nerves and upper limbs. However, he showed bilateral hip paraparesis with a power of $3 / 5$ on hip flexion and extension. His reflexes were $3+$ and his plantar responses were upgoing bilaterally. Sensory examination showed sensory level up to T7/T8. His EDSS score at presentation was three. Laboratory tests were normal. Brain and spine MRI revealed multiple periventricular and, to a lesser extent, subcortical white matter hyperintensities scattered within both cerebral hemispheres as well as an intramedullary hyperintense lesion at the level of T5 of the spine (Figure 3).

\section{Case 4}

A 32-year-old male had undergone bariatric surgery (bypass) in 2008. One year later after losing $35 \mathrm{~kg}$ (35lbs), he was referred to our neurology clinic with a history of numbness affecting his right fingertips, which later progressed to involve his right side of the body with dragging of the right foot that improved after one month. His symptoms were associated with urinary incontinence, constipation, and easy fatigability. Neurological examination revealed intact cranial nerves; however, power was $4 / 5$ in the upper limbs and left lower limb and $3 / 5$ in the right lower limb. Reflexes were 3+ with unsustained clonus in the right ankle. Plantar responses were upgoing bilaterally. Rapid alternating movements and fine finger movements were intact. He also showed right-sided hemiplegic gait. His EDSS score was three at presentation. MRI evidence heavily indicated MS (Figure 4).

\section{Case 5}

A 24-year-old female had undergone bariatric surgery (laparoscopic sleeve gastrectomy) in 2013. Three years after losing $59 \mathrm{~kg}$ (130lbs), she presented with a 2-3-week history of progressive abnormal posturing of the left hand and imbalance. Neurological examination revealed dystonic posturing of the left hand with dysmetria and dysdiadokokinesia on the left, indicative of basal ganglionic lesion or cerebellar degeneration. Her EDSS score was two at presentation. MRI revealed a lesion at the left pons (Figure 5).

The diagnosis of relapsing-remitting MS for all patients was performed based on the McDonald diagnostic criteria for RRMS (2010). Four patients underwent lumbar puncture (one denied consent) and indicated oligo-clonal bands.

\section{Discussion}

Currently, bariatric surgery is the most effective weight loss approach for treating obesity and its associated 
comorbidities due to its expeditious and long-lasting results. ${ }^{5}$ There have been reports associating it with various neurological symptoms affecting several neuraxis levels occurring early - less than one year after surgery - such as immediate peripheral nerve injury, encephalopathy, acute polyradiculoneuropathy, as well as of others occurring later such as optic neuropathy, peripheral neuropathy, and myopathy. ${ }^{12}$ In the current literature, there is no clear evidence of the relationship between bariatric surgery and MS; therefore, we present this case series to enhance clinician awareness and keep MS as a possible differential diagnosis for any neurological sequel occurring after this type of surgery not as a direct cause, but rather that can occur like any other disease.

Previous studies have reported that early-life obesity is associated with an increased risk of MS. However, a review article published in 2016 on the relationship between MS and obesity reported a general trend where obesity in adults aged 18-25 was associated with MS susceptibility whereas the association between childhood obesity and MS was less clear. ${ }^{13}$

There are two hypotheses on the underlying mechanism of this relationship. The first hypothesis suggests that obesity is associated with chronic low-grade inflammation and increased the production of proinflammatory adipocytokines. Inflammation in adipose tissue may occur as early as in childhood and the increased levels of Creactive protein and interleukin-6 indicate a proinflammatory response that may trigger $\mathrm{MS}^{13,14}$

All five patients in our case series were diagnosed with MS after bariatric surgery. The initial pathophysiological process for MS in these patients may be attributed to their obesity status. However, a retrospective case-control series study on the long-term surgical and ambulatory outcomes of previously severely obese MS patients after bariatric surgery reported an improvement in the MS patients who underwent the procedure compared with the control group. Moreover, there was a significant improvement in the quality of life among the MS patients who underwent the procedure, which was not observed in the control group. In addition, there were less acute MS exacerbations during the follow-up period in the MS patients who underwent the procedure compared with the control group ${ }^{15}$ and this may also simply tell us that every disease can occur after any surgery without direct relationship.

The second hypothesis involves the role of vitamin D. Adults and children with high body fat mass have lower circulating levels of vitamin D metabolites, resulting in an increased risk for MS and severe disease progression among Caucasians. Vitamin $D$ deficiency is one of the possible risk factors of $\mathrm{MS}^{13}$

Vitamin D plays an important role in calcium metabolism and bone homeostasis as well as in cellular regulation and differentiation. It is a potent immunomodulator in both the innate and adaptive immune system. A study on the immunomodulatory effect of vitamin $D$ reported that low levels of IL-4 and IL-12 in a group of MS patients were significantly increased by treatment with oral alfacalcidol (active vitamin D). Furthermore, altered bone metabolism in the MS patients suggested a relationship between vitamin $D$ and $\mathrm{MS}^{16}$

Several studies comparing vitamin D levels and sun exposure between MS patients and normal healthy individuals have reported lower vitamin D levels with no sunlight exposure in MS patients, indicating that sunlight exposure may reduce the risk of developing MS. In addition, clinical relapse phases and new MRI lesion appearances appear to occur more frequently during spring when vitamin $D$ storage and $1,25(\mathrm{OH}) 2 \mathrm{D} 3$ levels are at the lowest. $^{16}$

Another comprehensive study reported a greater prevalence of $\mathrm{MS}$ in regions with low ultraviolet exposure and that the risk of MS decreased after migration from higher to lower latitudes. ${ }^{17}$ We did not measure the levels of vitamin D prior to procedures; therefore, given that low vitamin $D$ levels could be a possible risk factor for MS, it could be a confounding variable in this report.

In addition, nutritional deficiency has been recognized as the general cause of the various late neurological complications following bariatric surgery. Commonly deficient nutrients include copper and zinc as well as vitamins such as thiamine (B1), cyanocobalamin (B12), folic acid, and vitamin $A .{ }^{18}$ In this report, we performed screening for zinc, copper, and vitamin B12 levels and excluded patients with nutritional deficiencies to rule out neurological symptoms caused by nutritional deficiency.

One study has reported a patient with MS-like syndrome due to a severe selenium deficiency. ${ }^{19}$ Although nutritional assessments for our five patients indicated normal results, we did not assess selenium levels. However, patients with nutritional deficiencies have been reported to experience repetitive attacks of vomiting or to fail to comply with their mineral and vitamin supplement regimens. ${ }^{5,6}$ None of our 
patients had any history of these symptoms.

Bitarafan et al. reported four patients who developed MS after severe intentional weight loss, which was attributed to result from an inflammatory process. ${ }^{7}$ Furthermore, four patients have been reported to develop systemic lupus erythematosus and rheumatoid arthritis, which are autoimmune diseases, after significant weight $\operatorname{loss}^{20}$ Our case series is consistent with these findings since all our patients were diagnosed with MS, which is also an autoimmune disease, after an average of two years of undergoing different bariatric surgeries.

Another case series reported three cases of MS after weight loss with one occurring due to bariatric surgery. One of the reported patients developed concomitant myasthenia gravis. $^{8}$ Surprisingly, all the three previously reported patients were males, which is similar to our report where four of our patients were males. This might indicate a genetic background since autoimmune diseases mostly occur in females.

It is imprudent to assert an obvious cause-effect relationship from the present cases. Therefore, we recommend that further studies with larger sample sizes and comprehensive nutritional assessments are performed to either validate or exile these findings.

\section{Conclusion}

There has been an increase in the number and variety of neurological complications following bariatric surgery, which corresponds with the increasing popularity and use of bariatric surgery. Any CNS part can be involved in these complications. Physicians should be familiar with the neurological disorders that may occur after this surgery when encountering such patients.

\section{References}

1. Flegal KM, Carroll MD, Kit BK. Prevalence of obesity and trends in the distribution of body mass index among US adults, 199-2010. JAMA. 2012;307(5):491-497.

2. Landais A. Neurological complications of bariatric surgery. Obes Surg. 2014;24(10):1800-1807.

3. Angrisani L, Santonicola A, lovino P, et al. Bariatric surgery worldwide 2013. Obes Surg. 2015;25:18221832.

4. Abarbanel JM, Berginer VM, Osimani A, et al. Neurologic complications after gastric restriction surgery for morbid obesity. Neurology. 1987;37:196-200.

5. Juhasz-Pocsine $K$, Rudnicki SA, Archer RL, et al. Neurologic complications of gastric bypass surgery for morbid obesity. Neurology. 2007;68:1843-1850.

6. Thaisetthawatkul P, Collazo-Clavell ML, Sarr MG, et al. A controlled study of peripheral neuropathy after bariatric surgery. Neurology. 2004;63:1462-1470.

7. Bitarafan S, Amani K, Sahraian M, et al. The first attack of multiple sclerosis presented immediately after voluntary and intensive weight loss: A case series. Iran J Neurol. 2017;16(1).

8. Alanazy $\mathrm{MH}$, Alomar MA, Aljafen $\mathrm{BN}$, et al. Multiple sclerosis and myasthenia gravis following severe weight loss. Neurosciences (Riyadh, Saudi Arabia). 2018;23(2):158-161.

9. Brownlee W, Hardy T, Fazekas F, et al. Diagnosis of multiple sclerosis: progress and challenges. Lancet. 2017;389(10076):1336-1346.

10. Polman C, Reingold S, Banwell B, et al. Diagnostic criteria for multiple sclerosis: 2010 Revisions to the McDonald criteria. Annals of Neurology. 2011;69(2):292-302.

11. Tabbara M, Carandina S, Bossi M, et al. Rare neurological complications after sleeve gastrectomy. Obes Surg. 2016;26(12):2843-2848.

12. Carvalho J, Medeiros C. Inflammatory polyneuropathy after bariatric surgery: report of two cases. J Neurol Disord. 2016;4(4).

13. Gianfrancesco MA, Barcellos LF. Obesity and multiple sclerosis susceptibility: a review. J Neurol Neuromedicine. 2016;1(7):1.

14. Matarese G, Procaccini C, De Rosa V. The intricate interface between immune and metabolic regulation: a role for leptin in the pathogenesis of multiple sclerosis? J Leukoc Biol. 2008;84:893-899.

15. Parikh MS, Shen $R$, Weiner $M$, et al. Laparoscopic bariatric surgery in super-obese patients (BMI> 50) is safe and effective: a review of 332 patients. Obes Surg. 2005;15(6):858-863.

16. Pierrot-Deseilligny C, Souberbielle JC. Vitamin D and multiple sclerosis: an update. Mult Scler Relat Disord. 2017;14:35-45.

17. Sintzel MB, Rametta $M$, Reder AT. Vitamin $D$ and multiple sclerosis: a comprehensive review. Neurology and Therapy. 2018;7(1):59-85.

18. Zafar A, Khatri IA. An overview of complications affecting the Central Nervous System following bariatric surgery. Neurosciences. 2018;23(2):4-12.

19. Algahtani $H$, Khan $A$, Khan $M$, et al. Neurological complications of bariatric surgery. Neurosciences. 2016;21(3):241-245.

20. Cañas CA, Echeverri AF, Ospina FE, et al. Is bariatric surgery a trigger factor for systemic autoimmune diseases? J Clin Rheumatol. 2016;22:89-91. 


\section{PEER REVIEW}

Not commissioned. Externally peer reviewed.

\section{CONFLICTS OF INTEREST}

The authors declare that they have no competing interests.

\section{FUNDING}

None

\section{PATIENT CONSENT}

The authors, Alshamrani F, Turkistani L, AlAmri N, AlZahrani $A$, Alsulaiman A, AlKhamis $F$, AlAbdali $M$, AlJaafari $D$, AlBaker A, AlAmri A, declare that:

1. They have obtained written, informed consent for the publication of the details relating to the patient(s) in this report.

2. All possible steps have been taken to safeguard the identity of the patient(s).

3. This submission is compliant with the requirements of local research ethics committees.

\section{Figures and Tables}

Figure 1: Axial brain MRI (FLAIR) showing periventricular oval-shaped hyper-intensities characteristic of MS

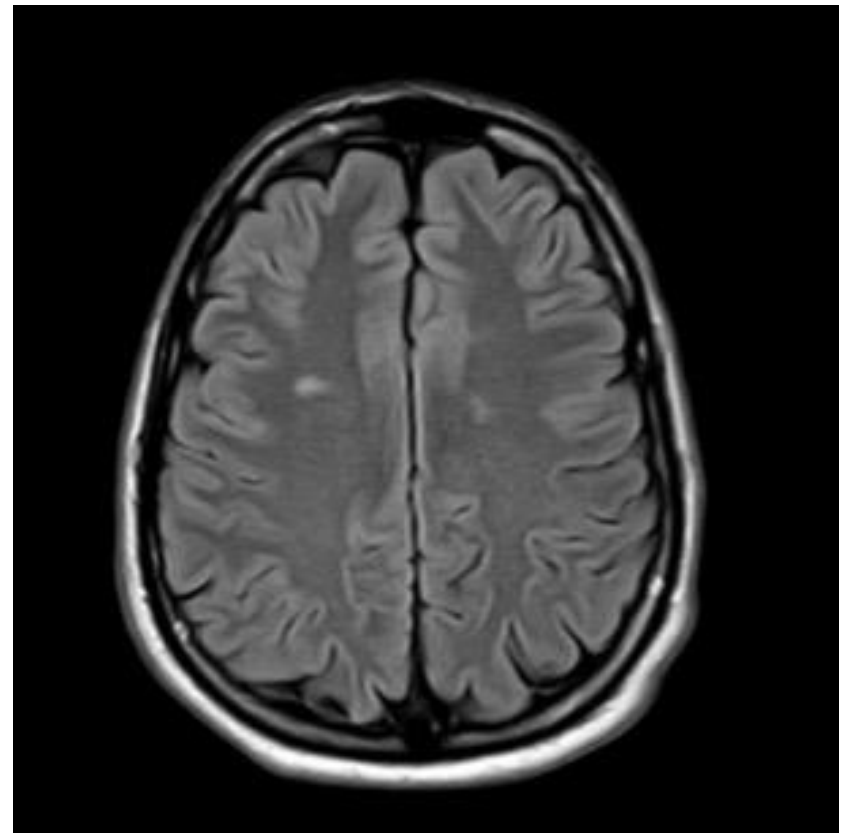

Figure 2: (A and B) axial brain MRI T2 and T1 with contrast showing enhanced right medulla oblongata lesions

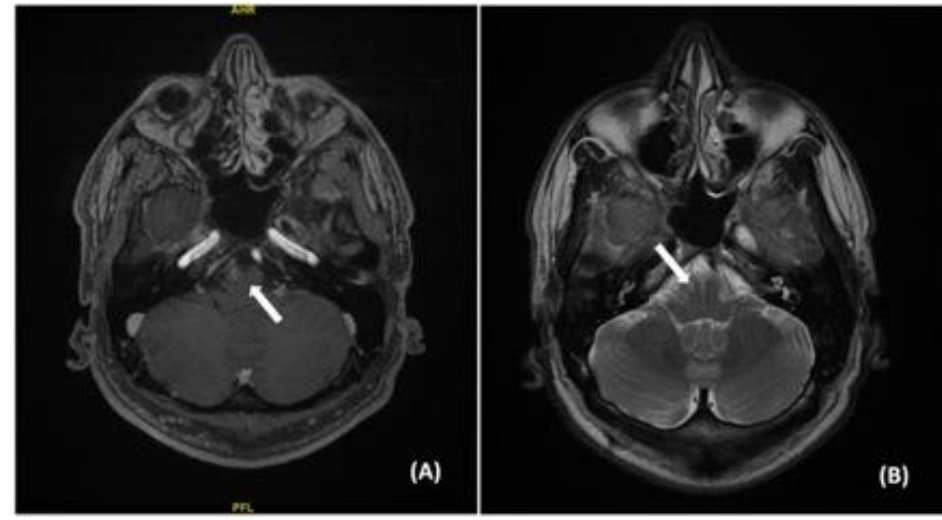

Figure 3: Sagittal cervical spine MRI (STAIR) showing intramedullary hyper-intensity at the level of T5 secondary to MS

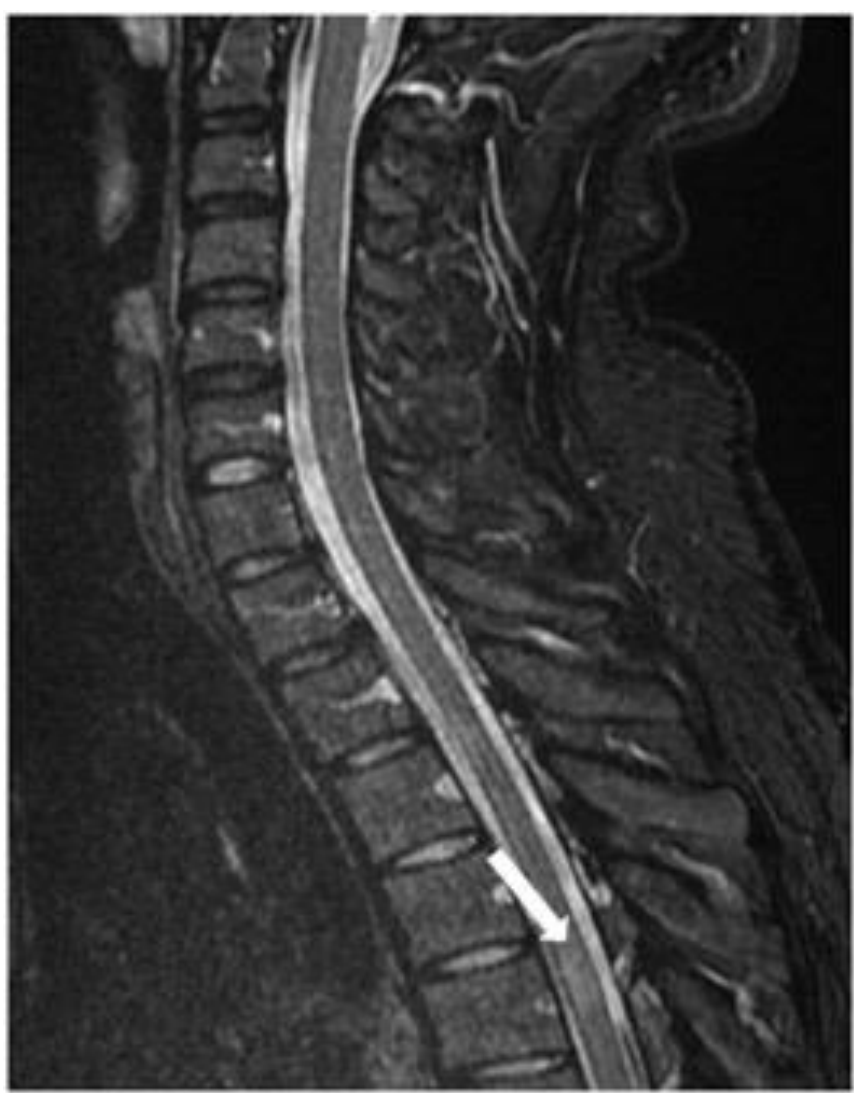


Figure 4: Axial brain MRI (FLAIR) showing juxtacortical and periventricular oval-shaped hyper-intensities characteristic of MS

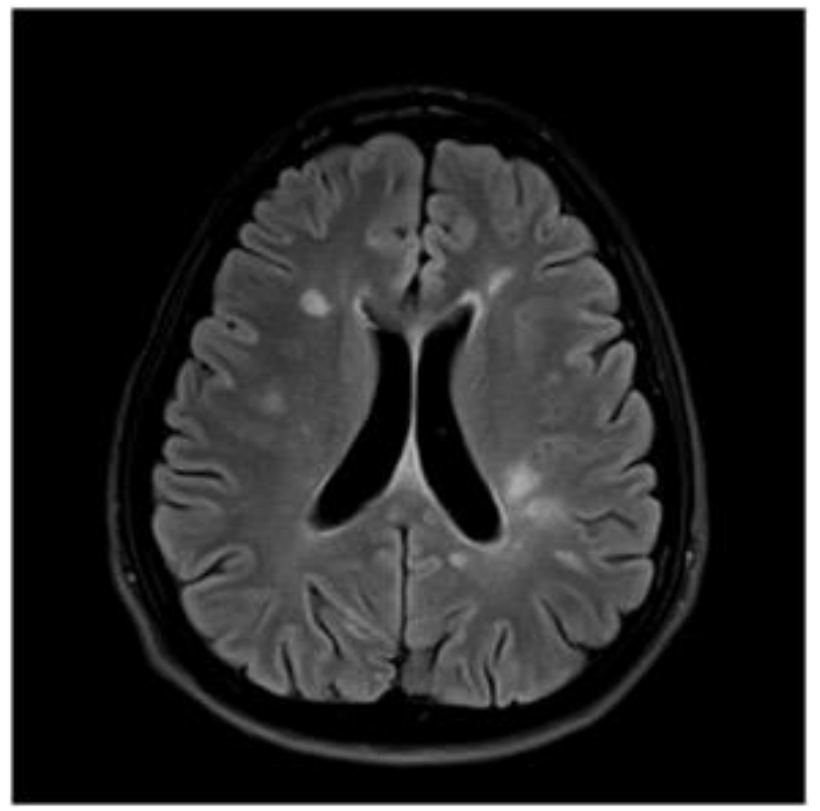

Figure 5: Axial brain MRI (T2) demonstrating high signal intensity at the left caudal ventral pons caused by MS

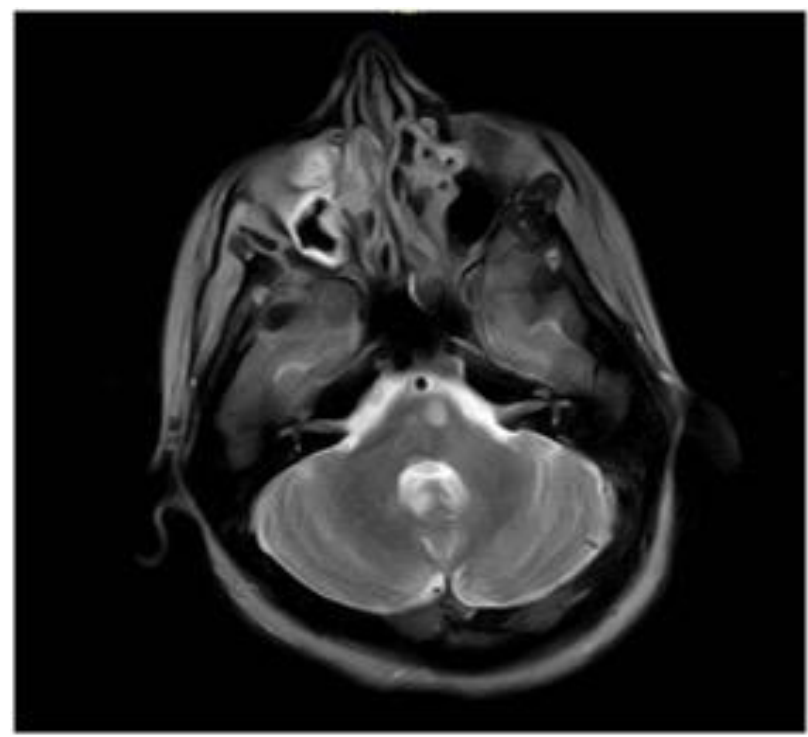


Table 1: Summary of the Cases

\begin{tabular}{|c|c|c|c|c|c|c|c|c|}
\hline \# & Sex & $\begin{array}{l}\text { Age } \\
\text { (years) }\end{array}$ & $\begin{array}{l}\text { BS } \\
\text { type }\end{array}$ & $\begin{array}{l}\text { Weight } \\
\text { loss (kg) }\end{array}$ & Clinical presentation & $\begin{array}{l}\text { EDSS } \\
\text { score }\end{array}$ & $\begin{array}{l}\text { Time to } \\
\text { presentation } \\
\text { (years) }\end{array}$ & CSF(OCB) \\
\hline 1 & $\widehat{0}$ & 31 & GB & 80 & ON & 3 & 3 & Negative \\
\hline 2 & 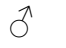 & 34 & LSG & 64 & Diplopia and TM & 3 & 1 & Positive \\
\hline 3 & $\widehat{0}$ & 29 & LSG & 56 & TM & 3 & 2 & Positive \\
\hline 4 & 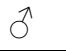 & 32 & GB & 35 & Numbness & 3 & 1 & Positive \\
\hline 5 & $q$ & 24 & LSG & 59 & Dystonia \& Numbness & 2 & 3 & Positive \\
\hline
\end{tabular}

BS, bariatric surgery; EDSS, expanded disability status scale; GB, gastric bypass; LSG, laparoscopic sleeve gastrectomy; ON, optic neuritis; TM, transverse myelitis; OCB, oligoclonal bands 